\title{
On the Statistics of Noisy Space Vector in Power Quality Analysis
}

\author{
Diego Bellan \\ Department of Electronics, Information and Bioengineering, Politecnico di Milano \\ Piazza Leonardo da Vinci 32, 20133 Milano, Italy \\ diego.bellan@polimi.it
}

\begin{abstract}
This work deals with the analysis of the impact of additive noise on the geometrical features of the space vector shape on the complex plane. The space vector shape is of great importance in the power quality analysis of modern three-phase power systems since its geometrical features are closely related to the voltage supply quality. In case of voltage sag or swell the space vector shape changes accordingly. In case of additive noise the geometrical parameters of the space vector shape can be treated as random variables. In the paper, the mean values and the variances of such parameters are derived in closed form as functions of the noise level and of the sampling conditions. Analytical results are validated through numerical simulation of the whole measurement process.
\end{abstract}

Keyword-Discrete Fourier transform, Noise propagation, Power quality, Power system analysis, Space vector, Statistical analysis

\section{INTRODUCTION}

Power quality is an issue of paramount importance in modern three-phase power systems where nonlinear and switching components result in voltage/current frequency spectra very rich in harmonics and interharmonics [1][5]. One of the main topics concerning power quality is the assessment of the voltage quality, i.e., the feature of the power system of maintaining the voltage supply at its nominal value. Within this context many papers have been published in the relevant literature concerning the monitoring of voltage level through the signal processing of the so-called space vector (e.g., [6]-[7]). The space vector is a complex valued function of time whose projections on proper axes provide the time behaviour of the three phase voltages. The reason why the space vector attracted the attention of many researchers is its capability of representing in a concise way the behaviour of three functions of time.

Signal processing of space vector has been especially related to the analysis of sags (i.e., dips) and swells of voltage supply in power systems [7]. The required analysis, however, consists in the evaluation of the geometrical parameters connected to the trajectory followed by the space vector on the complex plane. In fact, under steady-state conditions, the space vector follows an ellipse shape on the complex plane. Evaluation of voltage sags and swells requires an accurate determination of the axes and the inclination angle of the ellipse. As stated in [7], however, in many practical cases such evaluations are inaccurate due to the presence of harmonics/interharmonics and the contribution of corrupting noise. The impact of disturbing harmonics/interharmonics can be greatly reduced by means of a proper time-to-frequency transformation (i.e., the discrete Fourier transform) of the space vector. The impact of corrupting noise, however, can be still significant after transformation into the frequency domain. In the existing literature many papers have been devoted to the investigation of noise propagation through the discrete Fourier transform (e.g., see [8]-[17]). To the author's knowledge, however, the impact of noise on the determination of the geometrical parameters of the shape of space vector on the complex plane is still missing. The main contribution presented in this paper is the analytical investigation of the impact of corrupting noise on the determination of the features of the space vector shape on the complex plane. In particular, the statistical mean values and variances of the parameters of the space vector ellipse will be derived in closed form as functions of the input noise level and the sampling conditions.

The paper is organized as follows. In Section II the main definitions and properties concerning the space vector and its shape on the complex plane are recalled. Some properties related to the discrete Fourier transform of a complex valued function are also reported. In Section III the mean value and the variance of the parameters (i.e., the axes and the inclination angle) of the space vector ellipse are derived in closed form as functions of the input noise level and the sampling conditions (i.e., number of samples and the window function against spectral leakage). In Section IV numerical simulation are presented to validate the analytical results previously derived. Finally, conclusion remarks are presented in Section V. 


\section{Space Vector Definition and Power Quality Analysis}

Space vector is an effective tool for analysing three-phase power systems in time domain under both transient and steady-state conditions. Definition of space vector is based on the Clarke transformation [6]. Let us consider three phase voltages in time domain $v_{a}, v_{b}$, and $v_{c}$. The transformed voltages according to the Clarke transformation (in its rational form) are given by:

$$
\left[\begin{array}{l}
v_{\alpha} \\
v_{\beta} \\
v_{0}
\end{array}\right]=\sqrt{\frac{2}{3}}\left[\begin{array}{ccc}
1 & -\frac{1}{2} & -\frac{1}{2} \\
0 & \frac{\sqrt{3}}{2} & -\frac{\sqrt{3}}{2} \\
\frac{1}{\sqrt{2}} & \frac{1}{\sqrt{2}} & \frac{1}{\sqrt{2}}
\end{array}\right] \cdot\left[\begin{array}{l}
v_{a} \\
v_{b} \\
v_{c}
\end{array}\right]
$$

The voltage space vector is defined as a complex valued function with real and imaginary parts given by $\alpha$ and $\beta$ components, respectively:

$$
\vec{v}=v_{\alpha}+j v_{\beta}
$$

It can be readily proven that the straightforward formula to obtain the space vector from phase variables can be written as:

$$
\vec{v}=\sqrt{\frac{2}{3}}\left(v_{a}+a v_{b}+a^{2} v_{c}\right)
$$

where $a=\exp (j 2 \pi / 3)$. Under steady-state conditions (i.e., the working condition assumed in this paper) the space vector can be put into relation with positive and negative sequence variables obtained from the symmetrical component transformation (SCT) [18]-[19]. In fact, by considering the phasors $V_{a}, V_{b}$, and $V_{c}$ of the phase voltages at the frequency of interest (i.e., the fundamental or harmonic frequency), the sequence variables (i.e., positive, negative, and zero sequence voltages) according to the SCT (rational form) are given by:

$$
\left[\begin{array}{l}
V_{p} \\
V_{n} \\
V_{0}
\end{array}\right]=\frac{1}{\sqrt{3}}\left[\begin{array}{ccc}
1 & a & a^{2} \\
1 & a^{2} & a \\
1 & 1 & 1
\end{array}\right] \cdot\left[\begin{array}{c}
V_{a} \\
V_{b} \\
V_{c}
\end{array}\right]
$$

Thus, the relationship between the space vector (3) and the sequence voltages in (4) can be written as:

$$
\vec{v}=V_{p} \exp (j \omega t)+V_{n}^{*} \exp (-j \omega t)
$$

where asterisk denotes complex conjugate, and $\omega=2 \pi f$ is the angular frequency at the frequency $f$ of interest. Under general steady-state conditions the vector space (5) follows an ellipse shape on the complex plane. When the fundamental frequency is considered (i.e., 50 or $60 \mathrm{~Hz}$ for typical power systems), under ideal conditions the negative-sequence voltage $V_{n}$ is equal to zero and therefore the vector space follows a circle shape with radius $\left|V_{p}\right|$ on the complex plane.

As far as power quality is considered, it has been shown in the relevant literature that voltage dips and swells in a power system can be effectively characterized by analysing the shape of the space vector trajectory on the complex plane. In fact, under non-ideal conditions the space vector trajectory follows an ellipse shape whose parameters are of paramount importance in order to clearly identify the type of voltage dip or swell responsible for the change in space vector shape. More specifically, three key parameters of the space vector ellipse must be evaluated for power quality purpose, i.e., the major axis $R$, the minor axis $r$, and the inclination angle $\varphi$ of the ellipse (see Fig. 1). It can be shown that such parameters are given by [7]:

$$
\begin{gathered}
R=\left|V_{p}\right|+\left|V_{n}\right| \\
r=|| V_{p}|-| V_{n}|| \\
\varphi=\frac{1}{2}\left(\varphi_{p}-\varphi_{n}\right)
\end{gathered}
$$

where $\varphi_{p}$ and $\varphi_{n}$ are the angles of $V_{p}$ and $V_{n}$, respectively. As it was already pointed out in the relevant literature, practical estimation of the key parameters (6)-(8) can be greatly affected by noise due to both voltage waveforms corrupted by noise and measurement system superimposing additive noise. Therefore, a noisy space vector (see Fig. 2) results in inaccurate estimates of the key parameters (6)-(8) for power quality analysis [7].

Practical evaluation of $V_{p}$ and $V_{n}$ at fundamental frequency is usually performed by means of the discrete Fourier transform (DFT) of the space vector (3) [7]. In fact, evaluation of the spectral lines corresponding to the 
fundamental frequency allows to discard the disturbing contribution of harmonics, but the noise contribution cannot be cancelled and therefore it should be investigated. It is important here to recall that since the space vector (3) is a complex valued signal, the bilateral DFT spectrum must be considered because the spectral lines corresponding to negative frequencies are not the complex conjugate of the spectral lines for positive frequencies. In particular, when the fundamental frequency $f_{0}$ is considered, the DFT spectral line at $+f_{0}$ provides the positive sequence component $V_{p}$, whereas the DFT spectral line at $-f_{0}$ provides the negative (and complex conjugate) sequence component $V_{n}{ }^{*}$. Notice that in practical implementation of DFT a window function $w$ is used to reduce the well-known phenomenon called spectral leakage due to possible lack of synchronism between the waveform fundamental frequency and the sampling frequency [20]. Therefore, the estimates of positive and negative sequence components of the space vector (5) are obtained through the following formula:

$$
V_{m}=\frac{1}{N_{S} N P S G} \sum_{k=0}^{N_{S}-1} \overrightarrow{v_{k}} w_{k} \exp \left(-j 2 \pi m k / N_{S}\right)
$$

where NPSG is the Normalized Peak Signal Gain of the selected time window $w$ (e.g., NPSG is equal to 0.50 for the commonly used Hann window, whereas NPSG is equal to 1 for rectangular window), and $N_{S}$ is the number of samples of the waveform stretch. Notice that in (9) the space vector is given by (3) where each time domain phase voltage $v_{a}, v_{b}$, and $v_{c}$ is corrupted by additive noise. In the following analysis it is assumed that additive noise is white Gaussian independent noise with standard deviation $\sigma_{n}$.

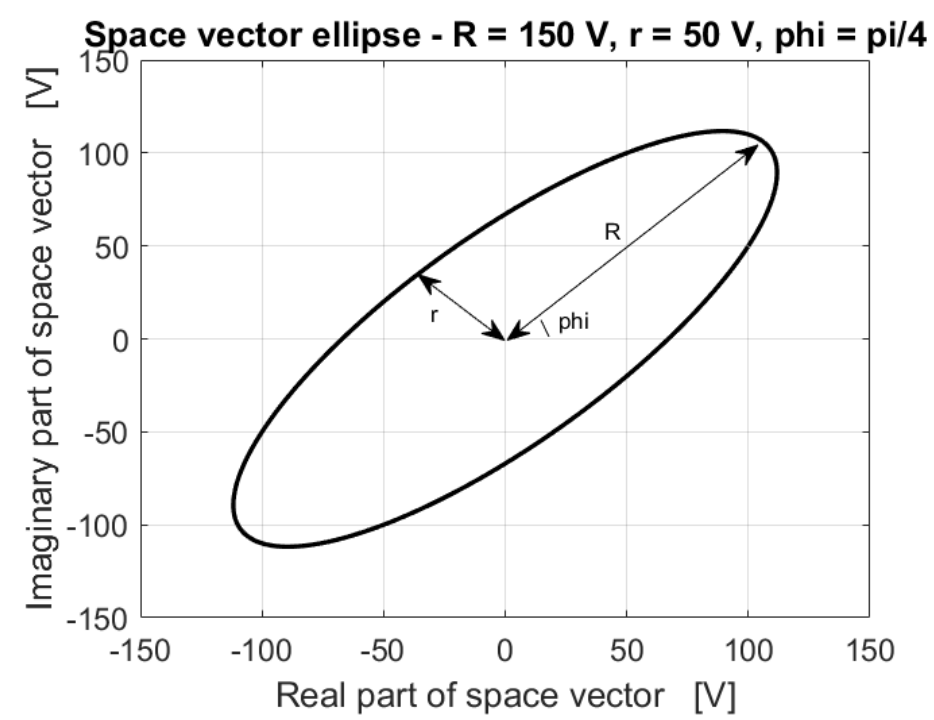

Fig. 1. Example of space vector following an ellipse shape with major axis $R=100 \mathrm{~V}$, minor axis $r=50 \mathrm{~V}$, and inclination angle $\varphi=\pi / 4$

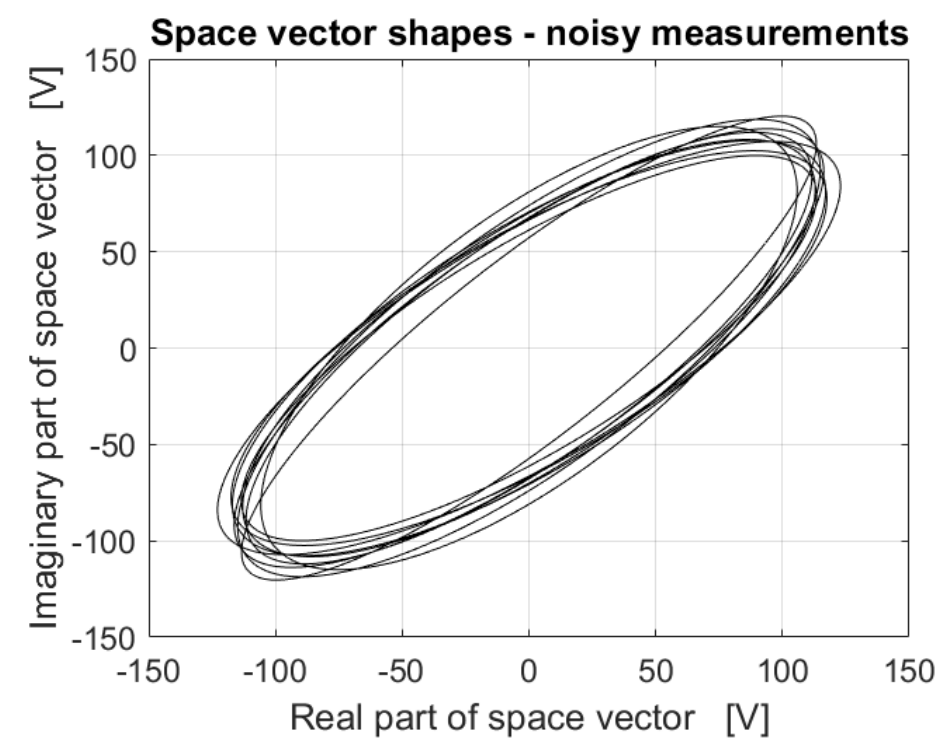

Fig. 2. Example of ten space vector shapes corresponding to ten different noisy measurements. Ellipse axes and inclination angle must be properly modelled as random variables. 


\section{Statistical Properties of Noisy Space Vector}

Propagation of additive noise through the DFT has been already thoroughly investigated in many papers in the past literature [8]-[17]. As far as the space vector and its use in power quality evaluations are concerned, from (6)-(8) it is clear that the statistical properties of positive and negative sequence voltages are needed. In [21] it was recently shown that the real and the imaginary parts of the negative sequence voltage can be effectively approximated by Gaussian and unbiased random variables (RVs) with variance

$$
\sigma^{2}=\frac{E N B W}{N_{S}} \sigma_{n}^{2}
$$

where ENBW is the Equivalent Noise Bandwidth of the window function $w$ used in (9) against spectral leakage (e.g., ENBW is equal to 1.50 for the Hann window, and equal to 1 for the rectangular window). The same results holds for the positive sequence voltage.

As far as the major and minor ellipse axes $R$ and $r$ in (6) and (7) are considered, from the above mentioned results we can readily observe that both $\left|V_{p}\right|$ and $\left|V_{n}\right|$ have a Rician distribution. Thus, the mean value of $R$ and $r$ can be expressed as follows [21]-[22]:

$$
\begin{aligned}
& \mu_{R}=\sigma \sqrt{\frac{\pi}{2}}\left[L_{1 / 2}\left(-\frac{\left|V_{p 0}\right|^{2}}{2 \sigma^{2}}\right)+L_{1 / 2}\left(-\frac{\left|V_{n 0}\right|^{2}}{2 \sigma^{2}}\right)\right] \\
& \mu_{r}=\sigma \sqrt{\frac{\pi}{2}}\left[L_{1 / 2}\left(-\frac{\left|V_{p 0}\right|^{2}}{2 \sigma^{2}}\right)-L_{1 / 2}\left(-\frac{\left|V_{n 0}\right|^{2}}{2 \sigma^{2}}\right)\right]
\end{aligned}
$$

where $V_{p 0}$ and $V_{n 0}$ are the noise-free values of the positive and negative sequence voltages, respectively, and $L$ is the Laguerre polynomial. Moreover, the variances of $R$ and $r$ are given by:

$$
\sigma_{R}^{2}=\sigma_{r}^{2}=4 \sigma^{2}+\left|V_{p 0}\right|^{2}+\left|V_{n 0}\right|^{2}-\frac{\pi \sigma^{2}}{2}\left[L_{1 / 2}^{2}\left(-\frac{\left|V_{p 0}\right|^{2}}{2 \sigma^{2}}\right)+L_{1 / 2}^{2}\left(-\frac{\left|V_{n 0}\right|^{2}}{2 \sigma^{2}}\right)\right]
$$

As far as the inclination angle $\varphi$ in (8) is considered, simple approximate formulas can be obtained for the mean value and the variance of $\varphi$ by means of the Taylor series approach [23]-[24]. To this aim, it can be reminded that the angles of the positive and negative sequence voltages are defined as:

$$
\varphi_{p, n}=\arctan \left(\frac{\operatorname{Im}\left(V_{p, n}\right)}{\operatorname{Re}\left(V_{p, n}\right)}\right)
$$

where, as mentioned above, the real and the imaginary parts are Gaussian unbiased RVs with variance (10). By taking into account only the first order terms in the Taylor expansion, the mean value of $\varphi$ is given simply by (8) evaluated in the noise-free case:

$$
\mu_{\varphi} \cong \frac{1}{2}\left(\varphi_{p 0}-\varphi_{n 0}\right)
$$

whereas the variance can be obtained by observing that for a RV $z$ expressed as a function of two RVs $x$ and $y$ as

$$
z=\arctan \left(\frac{y}{x}\right)
$$

the Taylor series approach provides:

$$
\sigma_{z}^{2} \cong\left|\frac{\partial z}{\partial x}\right|^{2} \sigma_{x}^{2}+\left|\frac{\partial z}{\partial y}\right|^{2} \sigma_{y}^{2}
$$

By using (17) in (14), after simple calculations the variance of (8) can be expressed as:

$$
\sigma_{\varphi}^{2} \cong \frac{1}{4} \sigma^{2}\left(\frac{1}{\left|V_{p 0}\right|^{2}}+\frac{1}{\left|V_{n 0}\right|^{2}}\right)
$$




\section{NUMERICAL VALIDATION}

Analytical results derived in Section III were validated through simulation of the measurement process of a noisy space vector. The three voltages were selected such that the noise-free positive sequence phasor was $V_{p 0}$ $=100 \mathrm{~V}$ and the negative sequence phasor $V_{n 0}=-j 50 \mathrm{~V}$. The corresponding phase voltages in the time domain (i.e., the three sinusoids at $50 \mathrm{~Hz}$ ), corrupted by additive white Gaussian noise with standard deviation $\sigma_{n}$, were sampled by taking $N_{S}=2^{12}$ samples in exactly 10 periods. The samples were windowed by means of a Hann window. A signal-to-noise ratio was defined as

$$
S N R=\frac{\sqrt{\left|V_{p 0}\right|^{2}+\left|V_{n 0}\right|^{2}}}{\sigma}
$$

where $\sigma$ was defined in (10). Different SNR values were selected by changing the value of $\sigma_{n}$ according to (10). For each specific value of SNR, $10^{3}$ repeated runs were performed for the numerical estimates of mean values and variances of the parameters of interest (i.e., the parameters (6)-(8)). Fig. 3 shows the behavior of the mean value of both the major and the minor axes defined in (6)-(7), normalized by the corresponding noise-free values, as functions of the SNR defined in (19). Dotted lines refer to numerical estimates through the simulation process outlined above, whereas solid lines represent the analytical results (11) and (12) properly normalized as mentioned above. Notice that both the axes are biased for low SNR. While the major axis $R$ has a positive bias, the minor axis $r$ has a negative bias. In any case the bias decreases as SNR increases. Fig. 4 compares numerical (dotted and dashed lines) with analytical (solid line) standard deviation (i.e., the square root of the variance) of the major and the minor axes as functions of SNR. According to (13), major and minor axes have the same standard deviation. The represented standard deviations are normalized with respect to $\sqrt{\left|V_{p 0}\right|^{2}+\left|V_{n 0}\right|^{2}}$. Finally, Fig. 5 shows the behavior of the standard deviation of the inclination angle defined in (8). Numerical results (dotted line) are compared with the analytical results in (18). It is interesting to notice that since (18) was obtained by means of an approximate approach based on Taylor expansion, the accuracy of the analytical results increase with SNR, i.e., the Taylor approach is obviously not effective for large noise levels, whereas its accuracy increases as the noise level decreases.

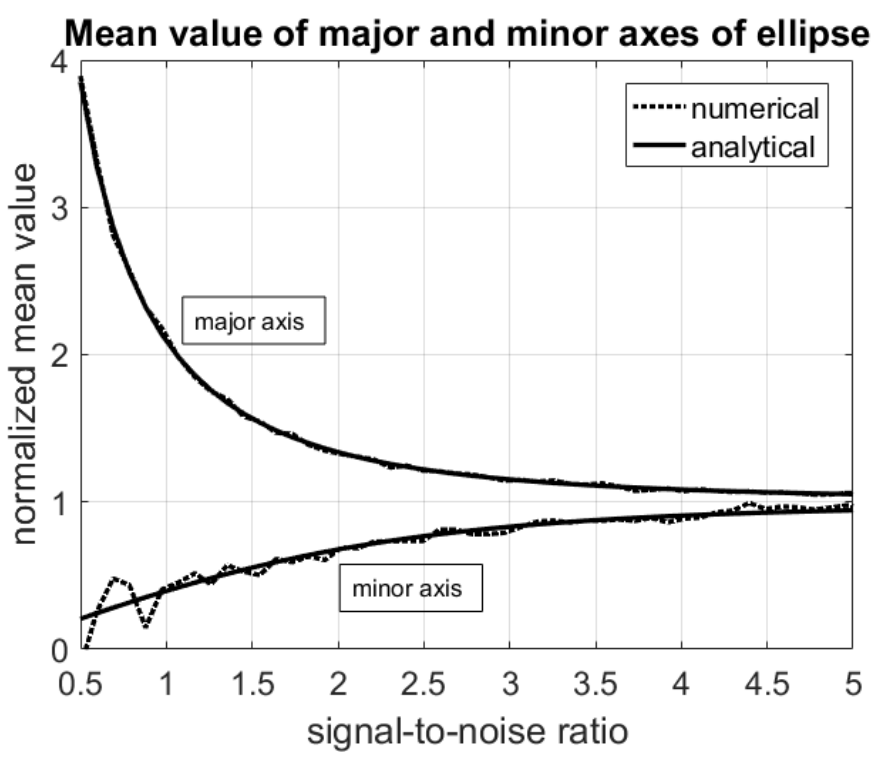

Fig. 3. Comparison between numerical and analytical behaviour of the normalized mean value of both the major and the minor axes of the space vector ellipse as functions of the SNR defined in (19). The mean values are normalized with respect to the corresponding noise-free values 


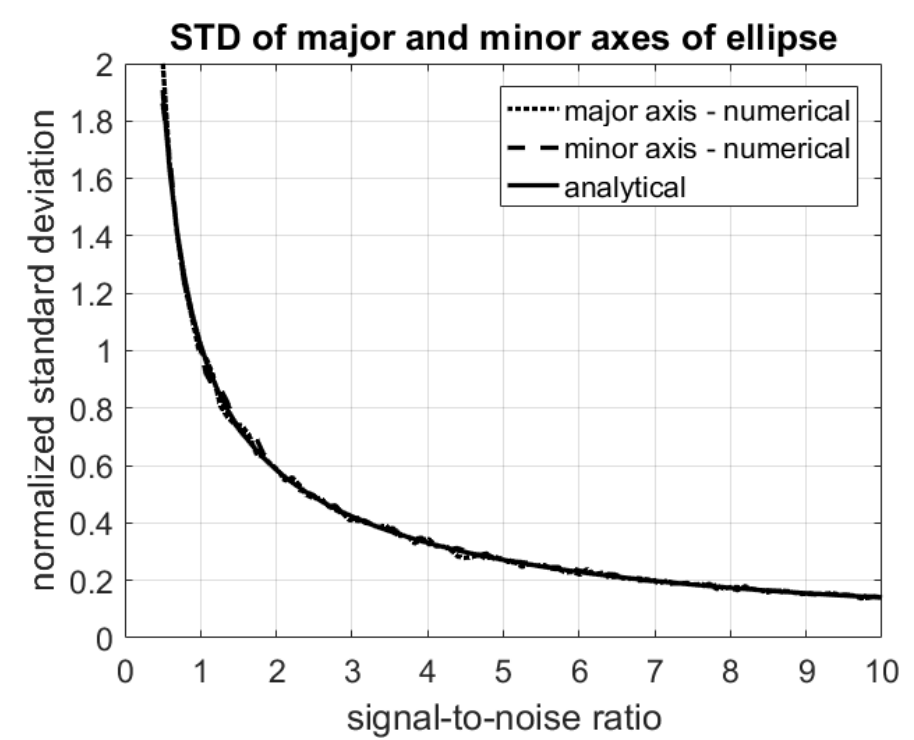

Fig. 4. Comparison between numerical and analytical behaviour of the normalized standard deviations of both the major and the minor axes of the space vector ellipse as functions of the SNR defined in (19)

\section{CONCLUSION}

The axes and the inclination angle of the space vector ellipse have been successfully modeled as random variables functions of the input noise level and of the sampling conditions. The analytical expressions derived in the paper for the mean values and the variances of such random variables proved to be accurate when validated against numerical simulations of the whole measurement process. In particular, the analytical results concerning the major and minor axes of the space vector ellipse proved to be very accurate. As far as the inclination angle was concerned, the analytical result for the variance was accurate only for higher SNR levels since the derivation was based on a Taylor expansion approach. The results presented in the paper are useful in order to quantify the uncertainty level in the evaluation of parameters related to voltage quality in modern threephase power systems.

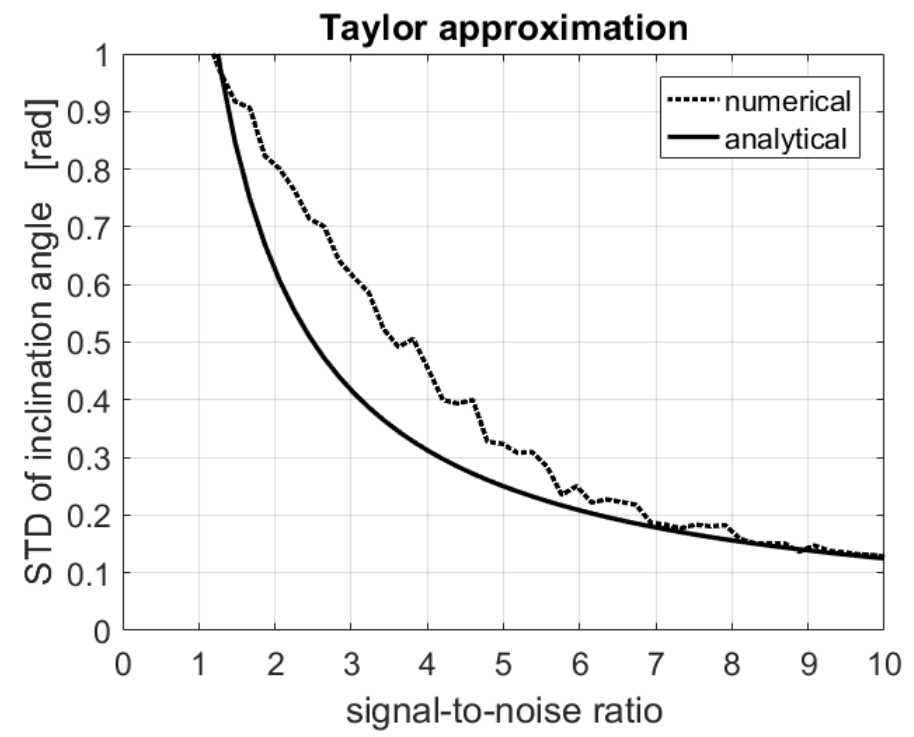

Fig. 5. Comparison between numerical and analytical behaviour of the standard deviation of the inclination angle of the space vector ellipse as functions of the SNR defined in (19). The analytical approximation (18) is based on the Taylor expansion and hence it provides increasing accuracy as the SNR increases

\section{REFERENCES}

[1] M. H. J. Bollen, Understanding Power Quality Problems, New York: IEEE Press, 2000

[2] A. Testa, M. F. Akram, R. Burch et al., "Interharmonics: Theory and Modeling," IEEE Trans. on Power Delivery, vol. 22, no. 4, pp. 2335-2348, Oct. 2007.

[3] M. Olofsson and U. Grape, "Voltage quality in the context of EMC," in Proc. International Symposium on Electromagnetic Compatibility, 2009, p. 241.

[4] D. Bellan, G, Spadacini, E. Fedeli, and S. A. Pignari, "Space-frequency analysis and experimental measurement of magnetic field emissions radiated by high-speed railway systems," IEEE Trans. Electromagn. Compat., vol. 55, no. 6, pp. 1031-1042, 2013. 
[5] D. Bellan, G. Superti-Furga, and S. A. Pignari, "Circuit representation of load and line asymmetries in three-phase power systems," International Journal of Circuits, Systems and Signal Processing, vol. 9, pp. 75-80, 2015.

[6] J. Aller, A. Bueno, and T. Paga, "Power system analysis using space vector transformation," IEEE Transactions on Power Systems, vol. 17, no. 4, pp. 957-965, November 2002.

[7] V. Ignatova, P. Granjon, and S. Bacha, "Space vector method for voltage dips and swells analysis," IEEE Trans. on Power Delivery, vol. 24, no. 4, pp. 2054 - 2061, 2009.

[8] O. M. Solomon, "The use of DFT windows in signal-to-noise ratio and harmonic distortion computations," IEEE Trans. Instrum. Meas., vol. 43, pp. 194-199, April 1994.

[9] D. Bellan, "Detection probability of harmonics in power systems affected by frequency fluctuation," International Journal of Circuits, Systems and Signal Processing, vol. 10, pp. 254-259, 2016.

[10] D. Bellan, "Estimation of Harmonic Power Components Affected by Frequency Instability," International Journal of Circuits, Systems and Signal Processing, vol. 10, pp. 275-280, 2016.

[11] D. Bellan, "Impact of Gaussian Frequency Deviation on the Detection Probability of Power System Harmonics," International Journal of Applied Engineering Research, vol. 11, no. 15, pp. 8515-8520, 2016.

[12] D. Bellan and S. A. Pignari, "Statistical Properties of Real-Time Amplitude Estimate of Harmonics Affected by Frequency Instability," Journal of Electrical Engineering (JEEEC), vol. 67, no. 4, pp. 292-298, 2016.

[13] D. Bellan, "Statistical Characterization of Harmonic Emissions in Power Supply Systems," International Review of Electrical Engineering, vol. 9, no. 4, pp. 803-810, 2014.

[14] D. Bellan, "Characteristic Function of Fundamental and Harmonic Active Power in Digital Measurements Under Nonsinusoidal Conditions," International Review of Electrical Engineering, vol. 10, no. 4, pp. 520-527, 2015.

[15] D. Bellan, A. Gaggelli, and S. A. Pignari, "Noise Effects in Time-Domain Systems Involving Three-Axial Field Probes for the Measurement of Nonstationary Radiated Emissions," IEEE Trans. on Electromagn. Compat., vol. 51, no. 2, pp. 192-203, 2009.

[16] D. Bellan, "Noise Propagation in Multiple-Input ADC-Based Measurement Systems," Measurement Science Review, vol. 14, no. 6, pp. 302-307, 2014

[17] D. Bellan, "On the Validity of the Noise Model of Quantization for the Frequency-Domain Amplitude Estimation of Low-Level Sine Waves," Metrology and Measurement Systems, vol. 22, no. 1, pp. 89-100, 2015.

[18] G. C. Paap, "Symmetrical components in the time domain and their application to power network calculations," IEEE Trans. on Power Systems, vol. 15 , no. 2 , pp. 522-528, May 2000

[19] D. Bellan, S. A. Pignari, G. Superti-Furga, "Consistent circuit technique for zero-sequence currents evaluation in interconnected single/three-phase power networks," Journal of Electrical Systems, vol. 12, no. 2, pp. 230-238, 2016.

[20] F. J. Harris, "On the use of windows for harmonic analysis with the discrete Fourier transform," Proc. of the IEEE, vol. 66, pp. 51-83, 1978.

[21] D. Bellan, "Statistical Properties of Voltage Unbalance Factor in Three-Phase Power Systems," International Journal of Applied Engineering Research, vol. 11, no. 15, pp. 8475-8479, Aug. 2016.

[22] M. K. Simon, Probability Distributions Involving Gaussian Random Variables, Springer, 2002.

[23] A. Papoulis and S. U. Pillai, Probability, Random Variables and Stochastic Processes, McGraw-Hill, 4th Ed., 2002.

[24] D. Bellan and S. A. Pignari, "Statistical superposition of crosstalk effects in cable bundles," China Communications, vol. 10, no. 11, pp. $119-128,2013$

\section{AUTHOR PROFILE}

Diego Bellan received the M.Sc. and the Ph.D. degrees in Electrical Engineering from the Politecnico di Milano, Milan, Italy, in 1994 and 1999, respectively. From June 1999 to August 2000 he was a Research Assistant with the Department of Electrical Engineering, Politecnico di Milano. He is currently an Assistant Professor of Advanced Circuit Theory with the Department of Electronics, Information and Bioengineering, Politecnico di Milano. His research interests are in the fields of electromagnetic compatibility (EMC), power quality, and analog-to-digital conversion of signals. He was involved in the staff of several funded research projects, concerning EMC in the space sector (European Space Agency) and in the railway sector (Trenitalia S.p.A.), and projects supported by the Italian Ministry of University and Research. He serves as reviewer for IEEE Transactions on Electromagnetic Compatibility, Instrumentation and Measurements, Industrial Electronics, and Industry Applications. 